\title{
3-亚甲基异苯并呋喃-1(3H)-酮及其衍生物合成新方法
}

\author{
王雅珍 $^{a}$ 朱星星 ${ }^{b}$ 林 伟 ${ }^{a}$ 郑纯智 ${ }^{a}$ 张继振 $*, a$ \\ ( ${ }^{a}$ 江苏理工学院化学与环境工程学院 常州 213001) \\ ( $b$ 常州大学石油化工学院 常州 213164)
}

\begin{abstract}
摘要 报道了 3-亚甲基异苯并呋喃-1 $(3 H)$-酮及其衍生物简易、高效的合成方法. 其特点是原料易得且价格低廉、反应 条件温和、收率高. 该方法以 2-甲基苯甲酸及其衍生物为原料, 经过酯化、自由基溴代和三苯基膦发生亲核取代反应 合成了 2-[(溴三苯基正膦基)甲基]苯甲酸甲酯及其衍生物; 随后发生 Wittig 反应、羧酸酯碱性条件下水解得到 2-乙烯基 苯甲酸及其衍生物; 接着和碘(NBS)发生环合反应, 生成 3-硔(溴)甲基异苯并呋喃-1(3H)-酮及其衍生物; 最后消除卤化 氢得到目标化合物.
\end{abstract}

关键词 3-亚甲基异苯并呋喃-1(3H)-酮; 3-磑(澳)甲基异苯并呋喃-1(3H)-酮; 衍生物; 季鏻盐; Wittig 反应; 合成

\section{A Novel Synthetic Method of 3-Methyleneisobenfuran- 1(3H)-one and Their Derivatives}

\author{
Wang, Yazhen ${ }^{a} \quad$ Zhu, Xingxing ${ }^{b} \quad$ Lin, Wei $^{a} \quad$ Zheng, Chunzhi ${ }^{a} \quad$ Zhang, Jizhen ${ }^{*, a}$ \\ ( ${ }^{a}$ School of Chemistry and Environmental Engineering, Jiangsu Technology of University, Changzhou 213001) \\ ( ${ }^{b}$ School of Petrochemical Engineering, Changzhou University, Changzhou 213164)
}

\begin{abstract}
A novel and highly efficient synthesis of 3-methyleneisobenzofuran-1(3H)-one and the corresponding derivatives was reported. The new method was characterized with raw material easy to get, low manufacturing cost, mild reaction conditions and high yields. 2-Methylbenzoic acid and their derivatives were used as starting material, methyl 2-((bromotri- phenylphosphoranyl)methyl)benzoate and their derivatives were synthesized through esterification, free-radical bromination with $\mathrm{N}$-bromobutanimide (NBS) and nucleophilic substitution with triphenylphosphine. After Wittig reaction and hydrolysis of carboxylic esters, 2-vinylbenzoic acid and their derivatives were obtained. Then through cyclization reaction with iodine (NBS), 3-iodo(bromo)methylisobenzofuran-1(3H)-one and their derivatives were obtained, which further react by elimination hydrogen halide with 1,8-diazabicyclo[5.4.0]under-7-ene to afford the target compounds. Eleven intermediates were new compounds.
\end{abstract}

Keywords 3-methyleneisobenzofuran-1(3H)-one; 3-iodo(bromo)methylisobenzofuran-1(3H)-one; derivatives; quaternary alkylphosphonium salt; Wittig reaction; synthesis

3-亚甲基异苯并呋喃-1(3H)-酮及其衍生物是由原 白头翁素(Protoanemonin)的环内双键与苯环或者取代苯 环稠合而成. 原白头翁素具有抗菌、抗肿瘤和杀虫等生 物活性 ${ }^{[1 ~ 6]}$, 并且认为原白头翁素中较大内应力的五元 环和亚甲基是具有生物活性的因素 ${ }^{[3]}$. 然而, 原白头翁 素的环外双键容易发生 $[2+2]$ 的环加成生成没有生物活 性的白头翁素(Anemonin)(图 1) ${ }^{[5,7]}$. 将原白头翁素的环 内双键和苯环或者取代苯环稠合, 保留环外双键(亚甲 基)和五元环的结构, 所得到的化合物如果具有和原白 头翁素类似的生物活性, 并且提高稳定性, 将具有重要<smiles>C=C1C=CC(=O)O1</smiles><smiles></smiles>

图 1 原白头翁素和白头翁素的构造式

Figure 1 Constitutional formulas of protoanemonin and anemonin

的意义. 考虑到苯环上取代基的类型和位置可能会影响 生物活性，本文设计合成 3-亚甲基异苯并呋喃-1(3H)-酮 及其衍生物。

\footnotetext{
*E-mail: zhangjizhen_9@aliyun.com
}

Received May 13, 2015; revised June 11, 2015; published online July 8, 2015 
3-亚甲基异苯并呋喃-1(3H)-酮的合成文献较多, 但 衍生物的合成文献较少. 3-亚甲基异苯并呋喃-1(3H)-酮 的合成除了前文综述的方法之外 ${ }^{[8,9]}$, 还有如下方法: 邻 乙炔基苯甲酸在(乙腈) [(2-联苯)二叔丁基膦]六氟锑酸 金(I)催化下, 发生分子内环合反应得到产物 ${ }^{[10]}$. 在 $\mathrm{Pd} / \mathrm{C}$ 、三苯基膦和碘化亚铜的催化体系下，邻溴苯甲酸 和三甲基硅基乙炔关环反应合成目标产物 ${ }^{[11]}$. 在催化剂 $\mathrm{CH}_{3} \mathrm{CO}_{2} \mathrm{M}(\mathrm{M}=\mathrm{Na} 、 \mathrm{~K} 、 \mathrm{Cs})$ 或者 $\mathrm{K}_{2} \mathrm{CO}_{3}$ 或者 $\mathrm{CH}_{3} \mathrm{CO}_{2} \mathrm{M} /$ $\mathrm{Al}_{2} \mathrm{O}_{3}$ 作用下, 邻苯二甲酸酐和乙酸高温反应 $\left(240{ }^{\circ} \mathrm{C}\right)$, 得到 3-亚甲基异苯并呋喃-1(3H)-酮 ${ }^{[12,13]}$. 上述合成方法 大多用到价格昂贵的催化剂, 有的用到重金属, 或者反 应条件苛刻(例如高温), 并且多数原料也需要合成. 为 了克服上述缺陷, 本研究小组以邻乙烯基苯甲酸甲酯及 其衍生物为原料, 水解之后进行环合, 接着发生消去反 应得到 3-亚甲基异苯并呋喃-1(3H)-酤及其衍生物 ${ }^{[8]}$. 由 于邻乙烯基苯甲酸甲酯及其衍生物是通过邻甲基苯甲 酸甲酯及其衍生物通过自由基溴代, 形成季鏻盐和发生 Wittig 反应制备的 ${ }^{[14]}$, 使得反应步骤较长. 我们又以原 料易得、价格低廉的异苯并呋喃-1(3H)-酮(苯酞)及其衍 生物为原料, 经过自由基溴代和三苯基膦发生亲核取代 反应得到季鏻盐, 接着发生消除反应生成 Wittig 试剂, 最后和甲醛发生 Wittig 反应得到 3-亚甲基异苯并呋喃$1(3 H)$-酮及其衍生物 ${ }^{[9]}$. 由于后二步反应产率较低, 有 必要继续探讨合成路线. 本文以 2-甲基苯甲酸及其衍生 物为原料, 采用新的合成方法, 经过以下反应较高产率 合成了 3 -亚甲基异苯并呋喃-1(3H)-酮及其衍生物 (Scheme 1), 为 3-亚甲基异苯并呋喃-1(3H)-酮及其衍生 物的合成提供了一条新途径.

\section{1 结果与讨论}

\subsection{2 -[(溴三苯基正膦基)甲基]苯甲酸甲酯及其衍生物} 4 的合成路线探讨

以 2-甲基苯甲酸及其衍生物为原料合成 2-[(溴三苯 基正膦基)甲基]苯甲酸甲酯及其衍生物 4, 需要三步反 应. 考虑到化合物 1 中的羧基可能和第二步反应中的 $N$ 溴代丁二酰亚胺(NBS)、第四步反应中的三乙胺反应, 首先把羧基转化成酯, 少量未反应的羧酸经过碳酸钠水 溶液洗涤除掉. 对于自由基溴代反应，控制 NBS 和化合 物 2 等物质的量, 从而避免了二溴代产物的生成. 反应 后 NBS 转化成丁二酰亚胺, 在四氯化碳中难溶, 抽滤除 去. 第三步反应生成的季鏻盐 4 在乙醚中难溶, 而少量 杂质易溶, 季鏻盐 4 的氯仿溶液加到适量的乙醚中, 4 析 出沉淀, 而杂质溶解在乙醚中. 三步反应总产率在 $69.5 \% \sim 85.2 \%$.

\subsection{3-碘甲基异苯并呋喃-1(3H)-酮及其衍生物 7 的合 成路线探讨}

邻乙烯基苯甲酸经间氯过氧苯甲酸环氧化得到 2环氧乙基苯甲酸, 然后在对甲苯磺酸催化下, 发生三元 环开环、同时再环合反应, 得到 3-羟甲基异苯并呋喃$1(3 H)$-酮 ${ }^{[8,14]}$. 3-差圣甲基异苯并呋喃-1(3H)-酮如果采用一 步法得到最终产物 3-亚甲基异苯并呋喃-1(3H)-酮, 需要 用强酸在高温下分子内脱水, 在这种条件下内酯易水 解, 难以得到产物. 文献 $[8,14]$ 采用了先把着基转化成 卤素或者 $\mathrm{TsO}$, 然后在碱的作用下脱卤化氢或者 $\mathrm{TsOH}$, 得到 3-亚甲基异苯并呋喃-1(3H)-酮及其衍生物，这种方 法步骤较多, 产率较低. 考虑到碘和碳碳双键亲电加成
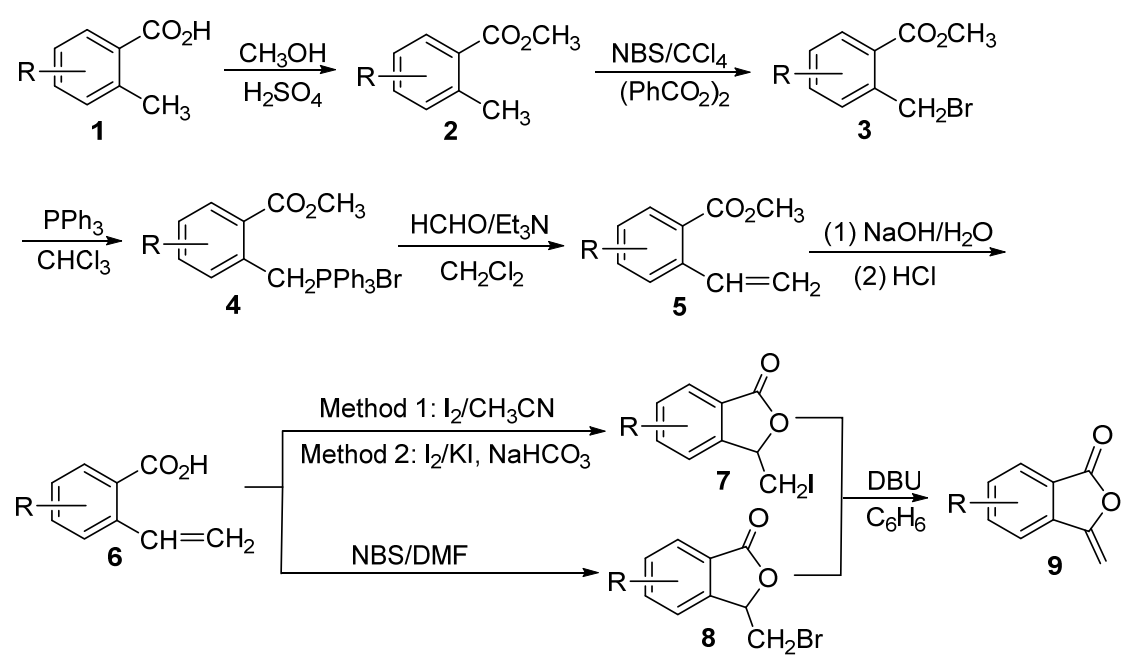

1a $\sim 9 \mathrm{a}: \mathrm{R}=\mathrm{H} ; 1 \mathrm{~b} \sim 6 \mathrm{~b}: \mathrm{R}=3-\mathrm{Br} ; \mathbf{7 b} \sim 9 \mathrm{~b}: \mathrm{R}=4-\mathrm{Br} ; 1 \mathrm{c} \sim 6 \mathrm{c}: \mathrm{R}=3-\mathrm{NO}_{2} ; \mathbf{7 c} \sim 9 \mathrm{c}: \mathrm{R}=4-\mathrm{NO}_{2} ; 1 \mathrm{~d} \sim 6 \mathrm{~d}$

$\mathrm{R}=4-\mathrm{Cl} ; \mathbf{7 d} \sim 9 \mathrm{~d}: \mathrm{R}=5-\mathrm{Cl}$; $1 \mathrm{e} \sim 6 \mathrm{ee}: \mathrm{R}=4-\mathrm{Br} ; 7 \mathrm{e} \sim 9 \mathrm{e}: \mathrm{R}=5-\mathrm{Br} ; 1 \mathbf{1 f} \sim 6 \mathrm{ff} \mathrm{R}=5-\mathrm{Br} ; \mathbf{7 f} \sim 9 \mathrm{f}: \mathrm{R}=6-\mathrm{Br}$

图式 13 -亚甲基异苯并呋喃-1(3H)-酮及其衍生物 9 的合成路线

Scheme 1 The synthetic route of 3-methyleneisobenzofuran-1(3H)-one and derivatives 9 
所形成的碘鎓离子比较稳定, 然后利用羧酸根阴离子和 三元环碘鎓离子发生分子内的 $\mathrm{S}_{\mathrm{N}} 2$ 反应，从而得到 3-碘 甲基异苯并呋喃-1(3H)-酮及其衍生物 7, 由于碘代物在 碱性条件下容易消除碘化氢, 得到最终产物. 与文献方 法相比 ${ }^{[8,14]}$, 本文采用的方法步骤少、产率较高. 环合反 应的可能机理 ${ }^{[15,16]}$ 如 Scheme 2: 邻乙烯基苯甲酸及其衍 生物 6 在碱性条件下形成羧酸根阴离子 $\mathbf{A}$, 碳碳双键与 单质碘发生亲电加成形成三元环的碘鎓离子 $\mathbf{B}$, 然后羧 酸根阴离子从三元环碘鎓离子的背面进攻碳原子, 发生 分子内的 $\mathrm{S}_{\mathrm{N}} 2$ 反应, 形成 3-碘甲基异苯并呋喃-1(3H)-酮 及其衍生物 7.

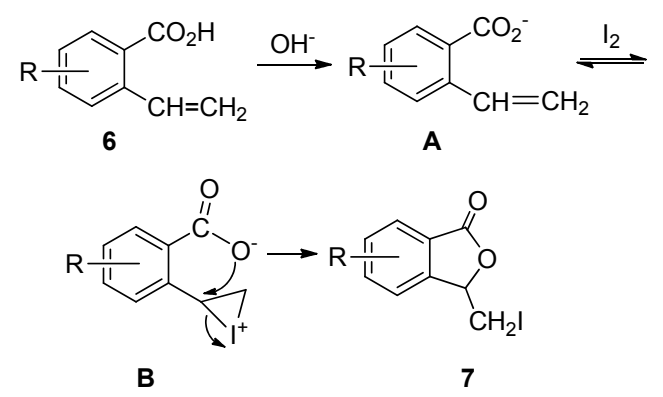

图式 2 环合反应机理

Scheme 2 The reaction mechanism of cyclization

3-碘甲基异苯并呋喃-1(3H)-酤及其衍生物 7 的合成 有两种方法: (1)将化合物 6 溶于乙腈中, 氮气保护下一 次性加入单质碘反应; (2)化合物 6 在碱性条件下逐滴加 入碘和碘化钾的水溶液, 合成 3-碘甲基异苯并呋喃$1(3 H)$-酮及其衍生物. 碘在水中的溶解度虽然很小, 但 在碘化钾溶液中溶解度却明显增大, 加碘化钾的目的是 增加单质碘在水中的溶解度. 两种方法的后处理相同, 收率见表 1 .

表 13 -碘甲基异苯并呋喃-1(3H)-酮及其衍生物 7 的合成 ${ }^{a}$

Table 1 Synthesis of 3-(iodomethyl)isobenzofuran-1(3H)-one and their derivatives 7

\begin{tabular}{|c|c|c|c|c|c|c|}
\hline \multirow{2}{*}{ Compd. } & \multirow{2}{*}{$\mathrm{R}$} & \multicolumn{2}{|c|}{ Method 1} & \multicolumn{2}{|c|}{ Method 2} & \multirow{2}{*}{ m.p. $/{ }^{\circ} \mathrm{C}$} \\
\hline & & Time/ & rield $^{b} / \%$ & Time/h & Yield $^{b} / \%$ & \\
\hline $7 a$ & $\mathrm{H}$ & 3 & 67.7 & 2 & 80.2 & $\begin{array}{l}69.2 \sim 70.7 \\
\left(67.6^{[17]}\right)\end{array}$ \\
\hline $7 b$ & $4-\mathrm{Br}$ & 6 & 68.5 & 3 & 71.4 & $133.6 \sim 135.8$ \\
\hline $7 \mathrm{c}$ & $4-\mathrm{NO}_{2}$ & 6 & 78.4 & 3 & 76.1 & $125.3 \sim 126.7$ \\
\hline $7 d$ & $5-\mathrm{Cl}$ & 6 & 90.0 & 3 & 75.9 & $130.4 \sim 134.3$ \\
\hline $7 e$ & $5-\mathrm{Br}$ & 6 & 90.4 & 3 & 80.6 & $140.7 \sim 142.2$ \\
\hline $7 f$ & $6-\mathrm{Br}$ & 6 & 94.9 & 3 & 75.2 & $115.2 \sim 115.9$ \\
\hline
\end{tabular}

a reaction condition: method 1: $\mathrm{I}_{2} / \mathrm{CH}_{3} \mathrm{CN}$, method 2: $\mathrm{I}_{2} / \mathrm{KI} / \mathrm{NaHCO}_{3} / \mathrm{H}_{2} \mathrm{O}$; ${ }^{b}$ Isolated yield.

\section{3 -溴甲基异苯并呋喃-1(3H)-酮及其衍生物 8 的合 成条件探索}

在化合物 7 的合成中用到了单质碘和碘化钾, 价格
都比较高，如果能用溴化物代替单质碘和碘化钾合成 3溴甲基异苯并呋喃-1(3H)-酮及其衍生物 8 将具有意义. 仅文献报道了 8 的合成方法 ${ }^{[18]}$ : 二氯甲烷为溶剂, 邻乙 烯基苯甲酸和 NBS 在手性催化剂 2,4-二甲氧基苯氨基 硫代甲酸奎尼丁酯催化下, 低温 $\left(-60{ }^{\circ} \mathrm{C}\right)$ 、避光、氮气 保护下反应 $4 \mathrm{~d}$, 粗产物柱层析得到 3-溴甲基异苯并呋 喃-1(3H)-酮.

以 6a 为原料, 本文研究了溶剂、溴化试剂和溴化试 剂用量对产率的影响. 试验中发现, 用 DMF 代替二氯 甲烷作溶剂，产率明显提高，而用乙醚和乙腈作溶剂， 产率非常低; 探讨了三种溴化试剂(单质液溴、单质液溴 与溴化钾配成的溶液、NBS)对产率的影响, 当以 NBS 作为溴化试剂、并且 $6 \mathbf{a}$ 和 NBS 的物质的量比 $1: 1.2$ 时收率最高, 在氮气保护下室温 $\left(25^{\circ} \mathrm{C}\right)$ 反应较理想, 3溴甲基异苯并呋喃-1(3H)-酮的产率 $89.8 \%$. 反应机理和 Scheme 2 类似.

\subsection{3-碘(溴)甲基异苯并呋喃-1(3H)-酮及其衍生物消 去反应条件探索}

化合物 7 消除碘化氢生成 3-亚甲基异苯并呋喃1(3H)-酮及其衍生物. 探讨了两种消除碘化氢的方法: 方法一以无水乙醇为溶剂, 用弱碱无水乙酸钠消除碘化 氢, 收率较高, 但是要求在绝对无水的环境下反应, 一 旦有水存在, 消除反应产率降低. 方法二以苯为溶剂, 用 1,8-二氮杂二环[5.4.0]十一碳-7-烯(DBU)有机碱消除 碘化氢, 反应无需绝对无水, 产率几乎是定量的, 故选 用方法二发生消除反应. 考察了 $\mathrm{DBU}$ 用量对产率的影 响，增加 DBU 的用量产率提高，但 DBU 和 $7 \mathbf{a}$ 的物质的 量比超过 1.83 时, 收率增加不明显, 故确定 DBU : 7a 的物质的量比为 1.83. DBU 是热敏性有机碱, 适当增加 反应温度能够提高反应收率. 试验发现反应温度 $80{ }^{\circ} \mathrm{C}$ 较理想. 化合物 8 消除溴化氢也存在类似的情况. 由于 消除碘化氢比消除溴化氢容易, 所以消除碘化氢的产率 更高.

\section{2 结论}

本文以原料易得、价格低廉的 2-甲基苯甲酸及其衍 生物为原料，采用新的合成路径，经过经典的有机反应 得到 6 个 3 -亚甲基异苯并呋喃-1(3H)-酮及其衍生物. 中 间体 2-[(溴三苯基正膦基)甲基]-4-氯苯甲酸甲酯(4d)是 新化合物，中间体 3-碘(溴)甲基异苯并呋喃-1(3H)-酮及 其衍生物 7 和 8 有 10 个是新化合物. 3-碘甲基异苯并呋 喃-1(3H)-酮及其衍生物 7 的合成采用二种方法: 方法一 是化合物 6 和单质碘在乙腈溶剂中反应，方法二是化合 物 6 在碱性条件下和单质碘的水溶液反应. $7 \mathrm{a}$ 和 $7 \mathrm{~b}$ 的产 率方法二高, 7c $\sim 7 \mathbf{f}$ 的产率方法一高. 探索了 3-溴甲基 
异苯并呋喃-1(3H)-酮及其衍生物 8 的合成工艺, $N, N^{\prime}$-二 甲基甲酰胺(DMF)为溶剂, 化合物 $\mathbf{6}$ 和 NBS 的物质的量 比为 $1: 1.2$, 氮气保护下室温反应 $6 \mathrm{~h}$, 产率最高. 3 -碘 (溴)甲基异苯并呋喃- 1(3H)-酮及其衍生物 7 和 8 易发生 消除 HX 的反应，高产率得到目标产物.

\section{3 实验部分}

\section{1 仪器与试剂}

WRS-1B 型数字显微熔点仪(上海精密科学仪器有 限公司), 温度未校正; AVANCE 500 III 型和 AVANCE400 III 超导傅里叶变换核磁共振波谱仪(瑞士 Bruker 公司), TMS 为内标; PROTÉGÉ 460 和 200 型傅 里叶红外光谱仪(美国 Nicolet 公司), 溴化钾压片法; 5975I 型质谱仪 (美国 Agilent 公司); 元素分析仪 EA2400 II(美国 PerkinElmer 公司). 试剂和溶剂均为化 学纯或分析纯, 柱层析采用 200 300 目硅胶(安徽良臣 硅源材料有限公司), 薄层层析采用青岛海洋化工厂生 产的 GF254 硅胶; 固体商业试剂除非特别说明外, 未经 纯化直接使用; 四氯化碳经五氧化二磷干燥蒸馏后使 用, 氯仿经氯化钙干燥蒸馏后使用.

\section{2 实验过程}

$2.2 .12-[$ (溴三苯基正膦基)甲基]苯甲酸甲酯及其衍 生物 4 合成通法

在 $250 \mathrm{~mL}$ 三口烧瓶中加入 $40 \mathrm{mmol}$ 化合物 1 和 80 $\mathrm{mL}$ 甲醇, 搅拌下室温缓慢滴加 $2 \mathrm{~mL}$ 浓硫酸, 滴完后加 热回流若干小时, TLC 跟踪反应进程[展开剂: $V$ (乙酸乙 酯) $: V($ 石油醚 $)=1: 4]$. 反应毕, 冷却至室温, 旋蒸除 去未反应的甲醇, 剩余物加入 $40 \mathrm{~mL}$ 无水乙醚, 有机相 分别用水、饱和碳酸钠溶液和食盐水 $(25 \mathrm{~mL} \times 2)$ 洗涤, 无水硫酸镁干燥有机相, 蒸去乙醚, 即得 2-甲基苯甲酸 甲酯及其衍生物 2. 将化合物 $\mathbf{2}$ 溶于 $30 \mathrm{~mL}$ 四氯化碳中, 搅拌下再加 $7.12 \mathrm{~g} \mathrm{(40} \mathrm{mmol} \mathrm{)} N$-溴代丁二酰胺亚胺以及 $0.51 \mathrm{~g}(2.1 \mathrm{mmol})$ 过氧化苯甲酰, 加热回流几小时, TLC 跟踪反应进程 [展开剂: $V$ (乙酸乙酯) $: ~ V($ 石油醚 $)=1$ : 4). 冷却至室温, 抽滤, 滤液分别用饱和亚硫酸氢钠溶 液、碳酸钠溶液和食盐水 $(25 \mathrm{~mL} \times 2)$ 洗涤, 无水硫酸镁 干燥, 减压蒸去四氯化碳, 即得 2-溴甲基苯甲酸甲酯及 其衍生物 3. 将化合物 3 溶于 $50 \mathrm{~mL}$ 氯仿中, 搅拌下加 入 $9.44 \mathrm{~g}(36 \mathrm{mmol})$ 三苯基膦, 控制温度在 $0 \sim 5{ }^{\circ} \mathrm{C}$ 反应 几小时, TLC 跟踪反应进程[展开剂: $V$ (乙酸乙酯) : $V$ (石 油醚 $)=1: 4]$. 氯仿溶液分别用饱和亚硫酸氢钠溶液、 饱和碳酸钠溶液和食盐水 $(25 \mathrm{~mL} \times 2)$ 洗涤, 将反应液缓 慢倒入 $100 \mathrm{~mL}$ 无水乙醚中, 析出大量固体, 放入冰箱 1 $\mathrm{h}$, 抽滤, 用无水乙醚 $(30 \mathrm{~mL} \times 4)$ 洗涤, 真空干燥得产物 (4).
2-[(溴三苯基正膦基)甲基]苯甲酸甲酯 $(\mathbf{4 a})^{[19,20]}$ : 白 色固体，产率 75.2\%. m.p. 230.4 231.2 ${ }^{\circ} \mathrm{C}$ (文献值 ${ }^{[21]}$ $\left.232 \sim 233{ }^{\circ} \mathrm{C}\right) ;{ }^{1} \mathrm{H}$ NMR $\left(400 \mathrm{MHz}, \mathrm{CDCl}_{3}\right) \delta: 7.81(\mathrm{t}, J=$ $8.0 \mathrm{~Hz}, 3 \mathrm{H}, \mathrm{ArH}), 7.68 \sim 7.63(\mathrm{~m}, 7 \mathrm{H}, \mathrm{ArH}), 7.57 \sim 7.52$ $(\mathrm{m}, 6 \mathrm{H}, \mathrm{ArH}), 7.48$ (d, $J=8.0 \mathrm{~Hz}, 1 \mathrm{H}, \mathrm{ArH}), 7.41 \sim 7.38$ (m, 1H, ArH), 7.07 (d, $J=8.0 \mathrm{~Hz}, 1 \mathrm{H}, \mathrm{ArH}), 5.31$ (d, $J=$ $\left.16.0 \mathrm{~Hz}, 2 \mathrm{H}, \mathrm{CH}_{2}\right), 3.60\left(\mathrm{~s}, 3 \mathrm{H}, \mathrm{CH}_{3}\right)$.

2-(溴三苯基正膦基)甲基-3-溴苯甲酸甲酯(4b) ${ }^{[22]}$. 白色固体，产率 $77.3 \%$. m.p. $216.3 \sim 217.6{ }^{\circ} \mathrm{C}$ (文献值 ${ }^{[22]}$ $\left.217.6 \sim 218.2{ }^{\circ} \mathrm{C}\right) ;{ }^{1} \mathrm{H}$ NMR $\left(400 \mathrm{MHz}, \mathrm{CDCl}_{3}\right) \delta: 7.82 \sim$ $7.63(\mathrm{~m}, 5 \mathrm{H}, \mathrm{ArH}), 7.68 \sim 7.63(\mathrm{~m}, 12 \mathrm{H}, \mathrm{ArH}), 7.32 \sim 7.28$ (m, 1H, ArH), 5.65 (s, 2H, $\left.\mathrm{CH}_{2}\right), 3.61\left(\mathrm{~s}, 3 \mathrm{H}, \mathrm{CH}_{3}\right)$.

2-(溴三苯基正膦基) 甲基-3-硝基苯甲酸甲酯 (4c) $)^{[23]}$ : 白色固体, 产率 69.5\%. m.p. 222.3 229.1 ${ }^{\circ} \mathrm{C}$ (文献值 ${ }^{[23]} 217 \sim 229{ }^{\circ} \mathrm{C}$ ); ${ }^{1} \mathrm{H} \mathrm{NMR}\left(400 \mathrm{MHz}, \mathrm{CDCl}_{3}\right) \delta$ : 8.09 (d, $J=7.6 \mathrm{~Hz}, 1 \mathrm{H}, \operatorname{ArH}), 7.96$ (d, $J=8.0 \mathrm{~Hz}, 1 \mathrm{H}$, ArH), $7.80 \sim 7.77(\mathrm{~m}, 3 \mathrm{H}, \mathrm{ArH}), 7.68 \sim 7.60(\mathrm{~m}, 13 \mathrm{H}$, ArH), 5.70 (s, 2H, $\left.\mathrm{CH}_{2}\right), 3.71\left(\mathrm{~s}, 3 \mathrm{H}, \mathrm{CH}_{3}\right)$.

2-(溴三苯基正膦基)甲基-4-氯苯甲酸甲酯(4d)：白 色固体，产率 77.6\%. m.p. 221.4 224.6 ${ }^{\circ} \mathrm{C} ;{ }^{1} \mathrm{H}$ NMR $\left(400 \mathrm{MHz}, \mathrm{CDCl}_{3}\right) \delta: 7.80 \sim 7.56(\mathrm{~m}, 5 \mathrm{H}, \mathrm{ArH}), 7.73 \sim$ $7.68(\mathrm{~m}, 6 \mathrm{H}, \mathrm{ArH}), 7.65 \sim 7.60(\mathrm{~m}, 6 \mathrm{H}, \mathrm{ArH}), 7.33(\mathrm{~d}, J=$ $8.8 \mathrm{~Hz}, 1 \mathrm{H}, \mathrm{ArH}), 6.15\left(\mathrm{~d}, J=15.2 \mathrm{~Hz}, 2 \mathrm{H}, \mathrm{CH}_{2}\right), 3.48$ (s, $\left.3 \mathrm{H}, \mathrm{CH}_{3}\right) ;{ }^{13} \mathrm{C} \mathrm{NMR}\left(100 \mathrm{MHz}, \mathrm{CDCl}_{3}\right) \delta: 165.79,139.62$, $135.12\left(\mathrm{~d},{ }^{3} J_{\mathrm{CCCP}}=3.0 \mathrm{~Hz}\right), 134.39\left(\mathrm{~d},{ }^{2} J_{\mathrm{CCP}}=9.8 \mathrm{~Hz}\right)$, $133.32,132.79,132.70,130.13\left(\mathrm{~d},{ }^{2} J_{\mathrm{CCP}}=12.6 \mathrm{~Hz}\right)$, $129.06,127.64,117.41\left(\mathrm{~d},{ }^{1} J_{\mathrm{CP}}=85.6 \mathrm{~Hz}\right), 52.25,28.59(\mathrm{~d}$, ${ }^{1} J_{\mathrm{CP}}=47.4 \mathrm{~Hz}$ ); IR (KBr) v: 3051, 2957, 2853, 1709, 1655, 1437, 1283, 1110, 891, $850 \mathrm{~cm}^{-1}$; MS (EI, $\left.70 \mathrm{eV}\right) \mathrm{m} / z(\%)$ : $355.2\left(\left[\mathrm{CH}_{2} \mathrm{PPh}_{3} \mathrm{Br}\right]^{+\cdot}, 2\right), 277.2\left(\left[\mathrm{CH}_{3} \mathrm{PPh}_{3}\right]^{+}, 80\right), 262.2$ $\left(\left[\mathrm{PPh}_{3}\right]^{+\cdot}, 100\right), 185.2\left(\left[\mathrm{M}+2-\mathrm{PPh}_{3} \mathrm{Br}\right]^{+}, 30\right), 183.2$ $\left(\left[\mathrm{M}-\mathrm{PPh}_{3} \mathrm{Br}\right]^{+}, 100\right), 169.1\left(\left[\mathrm{M}-\mathrm{CH}_{2} \mathrm{PPh}_{3} \mathrm{Br}\right]^{+}, 4\right)$. Anal. calcd for $\mathrm{C}_{27} \mathrm{H}_{23} \mathrm{BrClO}_{2} \mathrm{P}$ : C 61.68, $\mathrm{H} 4.41, \mathrm{Br} 15.20, \mathrm{Cl}$ 6.74, P 5.89; found $\mathrm{C} 61.57, \mathrm{H} 4.40, \mathrm{Br} 15.22, \mathrm{Cl} 6.75, \mathrm{P}$ 5.90 .

2-(溴三苯基正膦基)甲基-4-溴苯甲酸甲酯 $(4 \mathbf{e})^{[22]}$ : 白色固体，产率 80.2\%. m.p. 209.1 214.8 ${ }^{\circ} \mathrm{C}$ (文献值 ${ }^{[22]}$ $\left.206.8 \sim 218.5{ }^{\circ} \mathrm{C}\right) ;{ }^{1} \mathrm{H}$ NMR $\left(400 \mathrm{MHz}, \mathrm{CDCl}_{3}\right) \delta: 7.82 \sim$ $7.75(\mathrm{~m}, 4 \mathrm{H}, \mathrm{ArH}), 7.68 \sim 7.63(\mathrm{~m}, 12 \mathrm{H}, \mathrm{ArH}), 7.56(\mathrm{~d}, J=$ $8.0 \mathrm{~Hz}, 1 \mathrm{H}, \mathrm{ArH}), 7.40 \sim 7.36(\mathrm{~m}, 1 \mathrm{H}, \mathrm{ArH}), 5.62(\mathrm{~d}, J=$ $\left.16.0 \mathrm{~Hz}, 2 \mathrm{H}, \mathrm{CH}_{2}\right), 3.62\left(\mathrm{~s}, 3 \mathrm{H}, \mathrm{CH}_{3}\right)$.

2-(溴三苯基正膦基)甲基-5-溴苯甲酸甲酯(4f) ${ }^{[24]}$ : 白色固体，产率 78.9\%. m.p. 223 226.1 ${ }^{\circ} \mathrm{C}$ (文献值 ${ }^{[22]}$ 215 $\left.230{ }^{\circ} \mathrm{C}\right) ;{ }^{1} \mathrm{H}$ NMR (400 MHz, $\left.\mathrm{CDCl}_{3}\right) \delta: 7.99(\mathrm{~d}$, $J=2.0 \mathrm{~Hz}, 1 \mathrm{H}, \mathrm{ArH}), 7.89 \sim 7.87$ (m, 1H, ArH), 7.79 
$7.76(\mathrm{~m}, 3 \mathrm{H}, \mathrm{ArH}), 7.72 \sim 7.61(\mathrm{~m}, 13 \mathrm{H}, \mathrm{ArH}), 5.62(\mathrm{~d}, J=$ $\left.16.0 \mathrm{~Hz}, 2 \mathrm{H}, \mathrm{CH}_{2}\right), 3.62$ (s, 3H, $\mathrm{CH}_{3}$ ).

\subsubsection{2-乙烯基苯甲酸及其衍生物 6 合成通法}

在 $250 \mathrm{~mL}$ 三口烧瓶中加入 $25 \mathrm{mmol}$ 化合物 $\mathbf{4}$, 用 $100 \mathrm{~mL}$ 二氯甲烷溶解, 搅拌下一次性加入 $7.5 \mathrm{~g}(250$ $\mathrm{mmol}$ )多聚甲醛，氮气保护下缓慢滴加 $15 \mathrm{~mL}$ 三乙胺, 体系升温至 $80{ }^{\circ} \mathrm{C}$, 反应 $10 \mathrm{~h}$. 旋蒸除去 $50 \mathrm{~mL}$ 二氯甲 烷, 加入 $200 \mathrm{~mL}$ 石油醚, 析出大量三氯氧磷白色固体, 布氏漏斗放少量柱层析硅胶, 然后抽滤, 滤液中加入适 量的硅胶旋蒸至干, 然后硅胶柱层析[洗脱剂: $V$ (二氯甲 烷) $: V$ (石油醚 $)=2: 1]$, 层析液旋蒸, 真空干燥即得化

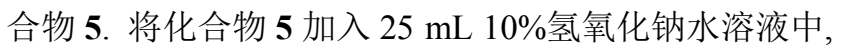
升温至 $70{ }^{\circ} \mathrm{C}$, 摚拌 $3 \mathrm{~h}$. 冷却至室温, 摚拌下向反应液 中缓慢滴加浓盐酸调至 $\mathrm{pH} \leqslant 2$, 析出白色固体, 抽滤, 用水洗涤，真空干燥得到化合物 $\mathbf{6}$.

2-乙烯基苯甲酸(6a $)^{[18,25]}$ : 白色固体, 产率 78.2\%. m.p. 92.6 93.5 ${ }^{\circ} \mathrm{C}$ (文献值 ${ }^{[25]} 94 \sim 95{ }^{\circ} \mathrm{C}$ ); ${ }^{1} \mathrm{H}$ NMR $(500$ $\left.\mathrm{MHz}, \mathrm{CDCl}_{3}\right) \delta: 8.06 \sim 8.04(\mathrm{~m}, 1 \mathrm{H}, \mathrm{ArH}), 7.62 \sim 7.54(\mathrm{~m}$, $3 \mathrm{H}, \operatorname{ArH}), 7.39 \sim 7.35(\mathrm{~m}, 1 \mathrm{H},=\mathrm{CH}), 5.68(\mathrm{dd}, J=$ $\left.17.5,1.0 \mathrm{~Hz}, 1 \mathrm{H},=\mathrm{CH}_{2}\right), 5.39(\mathrm{dd}, J=11.0,1.0 \mathrm{~Hz}, 1 \mathrm{H}$, $=\mathrm{CH}_{2}$ ).

2-乙烯基-3-溴苯甲酸 $(6 \mathbf{b})^{[26]}$ : 白色固体, 产率 76.2\%. m.p. $120.1 \sim 121.4{ }^{\circ} \mathrm{C}$ (文献值 ${ }^{[8]} 120.4 \sim$ $\left.120.6{ }^{\circ} \mathrm{C}\right) ;{ }^{1} \mathrm{H}$ NMR $\left(400 \mathrm{MHz}, \mathrm{CDCl}_{3}\right) \delta: 7.81 \sim 7.76(\mathrm{~m}$, $2 \mathrm{H}, \operatorname{ArH}), 7.21(\mathrm{t}, J=8.0 \mathrm{~Hz}, 1 \mathrm{H}, \operatorname{ArH}), 6.99 \sim 6.92(\mathrm{~m}$, $1 \mathrm{H},=\mathrm{CH}), 5.56\left(\mathrm{dd}, J=11.2,1.2 \mathrm{~Hz}, 1 \mathrm{H},=\mathrm{CH}_{2}\right), 5.39$ (dd, $J=18.0,1.2 \mathrm{~Hz}, 1 \mathrm{H},=\mathrm{CH}_{2}$ ).

2-乙烯基-3-硝基苯甲酸 $(\mathbf{6 c})^{[27]}$ : 白色固体, 产率 $74.2 \%$. m.p. $165.3 \sim 165.8{ }^{\circ} \mathrm{C}$ (文献值 $\left.{ }^{[28]} 165 \sim 166{ }^{\circ} \mathrm{C}\right)$; ${ }^{1} \mathrm{H}$ NMR $\left(400 \mathrm{MHz}, \mathrm{CDCl}_{3}\right) \delta: 8.19 \sim 8.15(\mathrm{~m}, 1 \mathrm{H}, \mathrm{ArH})$, $7.92 \sim 7.90(\mathrm{~m}, 1 \mathrm{H}, \mathrm{ArH}), 7.52(\mathrm{t}, J=8.0 \mathrm{~Hz}, 1 \mathrm{H}, \mathrm{ArH})$, $7.25 \sim 7.18(\mathrm{~m}, 1 \mathrm{H},=\mathrm{CH}), 5.46(\mathrm{~d}, J=11.6 \mathrm{~Hz}, 1 \mathrm{H},=$ $\left.\mathrm{CH}_{2}\right), 5.28\left(\mathrm{~d}, J=17.6 \mathrm{~Hz}, 1 \mathrm{H},=\mathrm{CH}_{2}\right)$.

2-乙烯基-4-氯苯甲酸 $(\mathbf{6 d})^{[29]}$ : 白色固体, 产率 76.7\%. m.p. $152.3{ }^{\circ} \mathrm{C}$ (熔点未见文献报道); ${ }^{1} \mathrm{H}$ NMR (400 $\left.\mathrm{MHz}, \mathrm{CDCl}_{3}\right) \delta: 7.99(\mathrm{~d}, J=8.4 \mathrm{~Hz}, 1 \mathrm{H}, \mathrm{ArH}), 7.58 \sim 7.51$ (m, 2H, ArH), $7.35 \sim 7.32(\mathrm{~m}, 1 \mathrm{H},=\mathrm{CH}), 5.69(\mathrm{~d}, J=$ $\left.17.6 \mathrm{~Hz}, 1 \mathrm{H},=\mathrm{CH}_{2}\right), 5.44\left(\mathrm{~d}, J=11.2 \mathrm{~Hz}, 1 \mathrm{H},=\mathrm{CH}_{2}\right)$.

2-乙烯基-4-澳苯甲酸 $(6 \mathbf{e})^{[8]}$ : 白色固体, 产率 77.7\%. m.p. $168.1 \sim 169.5{ }^{\circ} \mathrm{C}$ (文献值 ${ }^{[8]} 168.9 \sim$ $\left.170.4{ }^{\circ} \mathrm{C}\right) ;{ }^{1} \mathrm{H}$ NMR $\left(500 \mathrm{MHz}, \mathrm{CDCl}_{3}\right) \delta: 7.91$ (d, $J=8.5$ $\mathrm{Hz}, 1 \mathrm{H}, \mathrm{ArH}), 7.75$ (d , $J=2.0 \mathrm{~Hz}, 1 \mathrm{H}, \mathrm{ArH}), 7.56 \sim 7.49$ $(\mathrm{m}, 2 \mathrm{H}, \mathrm{ArH},=\mathrm{CH}), 5.68\left(\mathrm{~d}, J=17.5 \mathrm{~Hz}, 1 \mathrm{H},=\mathrm{CH}_{2}\right)$, $5.44\left(\mathrm{~d}, J=11.0 \mathrm{~Hz}, 1 \mathrm{H},=\mathrm{CH}_{2}\right)$.

2-乙烯基-5-溴苯甲酸 $(6 \mathbf{f})^{[8]}$ : 白色固体, 产率
75.2\%. m.p. $132 \sim 133.2{ }^{\circ} \mathrm{C}$ (文献值 ${ }^{[8]} 132.4 \sim 133.8{ }^{\circ} \mathrm{C}$ ); ${ }^{1} \mathrm{H}$ NMR $\left(400 \mathrm{MHz}, \mathrm{CDCl}_{3}\right) \delta: 8.17(\mathrm{~d}, J=2.0 \mathrm{~Hz}, 1 \mathrm{H}$, ArH), 7.66 (dd, $J=8.8,2.0 \mathrm{~Hz}, 1 \mathrm{H}, \mathrm{ArH}), 7.54 \sim 7.46(\mathrm{~m}$, $2 \mathrm{H}, \mathrm{ArH},=\mathrm{CH}), 5.68\left(\mathrm{~d}, J=17.6 \mathrm{~Hz}, 1 \mathrm{H},=\mathrm{CH}_{2}\right), 5.42$ (d, $J=10.8 \mathrm{~Hz}, 1 \mathrm{H},=\mathrm{CH}_{2}$ ).

\subsubsection{3-碘甲基异苯并呋喃-1 $(3 H)$-酮及其衍生物 7 合} 成通法

方法一: 在 $100 \mathrm{~mL}$ 三口烧瓶中加 $6.8 \mathrm{mmol}$ 化合物 (6), 加 $35 \mathrm{~mL}$ 乙腈溶解, 摚拌下加单质碘 $3.45 \mathrm{~g}$ (13.6 $\mathrm{mmol})$, 氮气保护, 室温反应几小时, TLC 跟踪反应进程 [展开剂: $V$ (乙酸乙酯) $: V($ 石油醚 $)=1 ： 4$ ]. 向反应液加 $30 \mathrm{~mL}$ 饱和硫代硫酸钠溶液, 搅拌均匀后, 用乙酸乙酯 $(30 \mathrm{~mL} \times 3)$ 萃取, 有机层分别用饱和碳酸氢钠溶液、硫 代硫酸钠溶液和食盐水 $(25 \mathrm{~mL} \times 2)$ 洗涤, 无水硫酸镁干 燥, 旋蒸除掉乙酸乙酯和乙腈, 残余物乙醇重结晶, 真 空干燥得化合物 7.

方法二: 在 $250 \mathrm{~mL}$ 三颈瓶中加入 $6 \mathrm{mmol}$ 化合物 $\mathbf{6}$, 加 $60 \mathrm{~mL}$ 的饱和碳酸氢钠溶液使其溶解, 摚拌均匀后滴 加 $\mathrm{I}_{2}$ 和 $\mathrm{KI}$ 的水溶液 $\left(1.50 \mathrm{~g}\right.$ 的 $\mathrm{I}_{2}$ 和 $3.60 \mathrm{~g}$ 的 $\mathrm{KI}$ 溶于 30 $\mathrm{mL}$ 水中), 室温反应几小时, TLC 跟踪反应进程[展开剂: $V($ 乙酸乙酯 $): V($ 石油醚 $)=1: 4]$. 向反应液加 $30 \mathrm{~mL}$ 饱 和硫代硫酸钠溶液, 搅拌均匀后, 用乙酸乙酯 (30 $\mathrm{mL} \times 3$ )萃取, 有机层分别用饱和碳酸氢钠溶液、饱和硫 代硫酸钠溶液和饱和食盐水 $(25 \mathrm{~mL} \times 2)$ 洗涤, 无水硫酸 镁干燥，旋蒸除掉乙酸乙酯，残余物乙醇重结晶，真空 干燥得化合物 7.

3-碘甲基异苯并呋喃-1(3H)-酮(7a) ${ }^{[30]}$ : 淡黄色固体. ${ }^{1} \mathrm{H}$ NMR $\left(400 \mathrm{MHz}, \mathrm{CDCl}_{3}\right) \delta: 7.94(\mathrm{~d}, J=8.0 \mathrm{~Hz}, 1 \mathrm{H}$, $\mathrm{ArH}), 7.75 \sim 7.71(\mathrm{~m}, 1 \mathrm{H}, \mathrm{ArH}), 7.62 \sim 7.59(\mathrm{~m}, 2 \mathrm{H}, \mathrm{ArH})$, $5.49(\mathrm{t}, J=4.8 \mathrm{~Hz}, 1 \mathrm{H}, \mathrm{CH}), 3.65(\mathrm{dd}, J=11.2,5.6 \mathrm{~Hz}, 1 \mathrm{H}$, $\mathrm{CH}_{2}$ ), 3.59 (dd, $J=11.2,4.8 \mathrm{~Hz}, 1 \mathrm{H}, \mathrm{CH}_{2}$ ).

3-碘甲基-4-溴异苯并呋喃-1(3H)-酮(7b): 淡黄色固 体. ${ }^{1} \mathrm{H}$ NMR $\left(400 \mathrm{MHz}, \mathrm{CDCl}_{3}\right) \delta: 7.90(\mathrm{~d}, J=7.6 \mathrm{~Hz}, 1 \mathrm{H}$, $\operatorname{ArH}), 7.83$ (d, $J=7.6 \mathrm{~Hz}, 1 \mathrm{H}, \operatorname{ArH}), 7.50$ (t, $J=8.0 \mathrm{~Hz}$, $1 \mathrm{H}, \mathrm{ArH}), 5.38$ (t, $J=3.6 \mathrm{~Hz}, 1 \mathrm{H}, \mathrm{CH}), 4.04$ (dd, $J=11.6$, $\left.4.4 \mathrm{~Hz}, 1 \mathrm{H}, \mathrm{CH}_{2}\right), 3.92\left(\mathrm{dd}, J=11.6,3.2 \mathrm{~Hz}, 1 \mathrm{H}, \mathrm{CH}_{2}\right) ;{ }^{13} \mathrm{C}$ NMR $\left(100 \mathrm{MHz}\right.$, DMSO- $\left.d_{6}\right) \delta: 167.86,147.63,137.87$, 132.21, 128.73, 124.44, 116.05, 78.28, 7.21; IR (KBr) $v$ : 3448, 1763, 1458, 1303, 1256, 1068, 964, 758, $619 \mathrm{~cm}^{-1}$; MS (EI, $70 \mathrm{eV}) \mathrm{m} / \mathrm{z}(\%): 353.9\left(\left[\mathrm{M}^{+\cdot}+2\right], 21\right), 351.9$ ([M $\left.\left.\mathrm{M}^{+\cdot}\right], 21\right), 227.0\left([\mathrm{M}+2-\mathrm{I}]^{+}, 69\right), 225.0\left([\mathrm{M}-\mathrm{I}]^{+}, 66\right)$, $213.0\left(\left[\mathrm{M}+2-\mathrm{CH}_{2} \mathrm{I}\right]^{+}, 71\right), 211.0\left(\left[\mathrm{M}-\mathrm{CH}_{2} \mathrm{I}\right]^{+}, 68\right)$, $185.0\left(\left[m-\mathrm{BrC}_{6} \mathrm{H}_{4} \mathrm{CO}+2\right]^{+}, 19\right), 183.0\left(\left[m-\mathrm{BrC}_{6} \mathrm{H}_{4} \mathrm{CO}\right]^{+}\right.$, 17), $182.0\left(\left[\mathrm{M}-\mathrm{ICH}_{2} \mathrm{CHO}\right]^{+}, 3\right), 155.0\left(\left[m-\mathrm{BrC}_{6} \mathrm{H}_{4}\right]^{+}\right.$, 11), $127.0\left(\left[\mathrm{I}^{+}\right], 13\right), 104.1\left(\left[\mathrm{M}-\mathrm{Br}-\mathrm{CH}_{2} \mathrm{I}-\mathrm{CO}\right]^{+*}, 7\right)$. 
Anal. calcd for $\mathrm{C}_{9} \mathrm{H}_{6} \mathrm{BrIO}_{2}$ : C 30.63, $\mathrm{H} \mathrm{1.71,} \mathrm{Br} 22.64$, I 35.96; found C 30.59, H 1.71, Br 22.67, I 35.99.

3-碘甲基-4-硝基异苯并呋喃-1(3H)-酮(7c): 淡黄色 固体. ${ }^{1} \mathrm{H}$ NMR $\left(400 \mathrm{MHz}, \mathrm{CDCl}_{3}\right) \delta: 8.55(\mathrm{~d}, J=8.0 \mathrm{~Hz}$, $1 \mathrm{H}, \mathrm{ArH}), 8.30$ (d, $J=7.6 \mathrm{~Hz}, 1 \mathrm{H}, \operatorname{ArH}), 7.87$ (t, $J=8.0$ $\mathrm{Hz}, 1 \mathrm{H}, \mathrm{ArH}), 6.01(\mathrm{t}, J=3.2 \mathrm{~Hz}, 1 \mathrm{H}, \mathrm{CH}), 4.07$ (dd, $J=$ 11.6, $3.6 \mathrm{~Hz}, 1 \mathrm{H}, \mathrm{CH}_{2}$ ), $3.92(\mathrm{dd}, J=11.6,3.2 \mathrm{~Hz}, 1 \mathrm{H}$, $\left.\mathrm{CH}_{2}\right) ;{ }^{13} \mathrm{C}$ NMR (100 MHz, DMSO- $\left.d_{6}\right) \delta: 167.00,143.23$, $142.94,132.42,131.70,129.96,129.61,79.33,7.30$; IR (KBr) v: 3105, 2925, 1782, 1466, 1309, 1078, 1027, 969, 748, $600 \mathrm{~cm}^{-1}$; MS (EI, $\left.70 \mathrm{eV}\right) \mathrm{m} / z(\%): 320.0\left(\left[\mathrm{M}^{+\cdot}+1\right]\right.$, 24), $319.0\left(\left[\mathrm{M}^{+\cdot}\right], 1\right), 192.1\left([\mathrm{M}-\mathrm{I}]^{+}, 100\right), 178.1([\mathrm{M}-$ $\left.\left.\left.\mathrm{CH}_{2} \mathrm{I}\right]^{+}, 37\right), 149.2\left(\left[\mathrm{M}-\mathrm{ICH}_{2} \mathrm{CHO}\right]^{+\cdot}, 2\right), 127.0\left(^{(} \mathrm{I}^{+}\right], 4\right)$, $104.1\left(\left[\mathrm{M}-\mathrm{NO}_{2}-\mathrm{CH}_{2} \mathrm{I}-\mathrm{CO}\right]^{+}\right.$, , 23). Anal. calcd for $\mathrm{C}_{9} \mathrm{H}_{6} \mathrm{INO}_{4}$ : C 33.88, H 1.90, I 39.78, N 4.39; found $\mathrm{C}$ 33.84, H 1.90, I 39.82, N 4.40.

3-碘甲基-5-氯异苯并呋喃-1(3H)-酮(7d): 淡黄色固 体. ${ }^{1} \mathrm{H}$ NMR $\left(400 \mathrm{MHz}, \mathrm{CDCl}_{3}\right) \delta: 7.86(\mathrm{~d}, J=8.0 \mathrm{~Hz}, 1 \mathrm{H}$, ArH), $7.62 \sim 7.57(\mathrm{~m}, 2 \mathrm{H}, \mathrm{ArH}), 5.46(\mathrm{t}, J=5.2 \mathrm{~Hz}, 1 \mathrm{H}$, $\mathrm{CH}), 3.62\left(\mathrm{dd}, J=10.8,5.2 \mathrm{~Hz}, 1 \mathrm{H}, \mathrm{CH}_{2}\right), 3.58(\mathrm{dd}, J=$ 10.8, $\left.4.4 \mathrm{~Hz}, 1 \mathrm{H}, \mathrm{CH}_{2}\right) ;{ }^{13} \mathrm{C} \mathrm{NMR}\left(100 \mathrm{MHz}, \mathrm{CDCl}_{3}\right) \delta$ : $168.17,149.83,141.09,130.79,127.04,125.11,122.77$, 77.90, 5.05; IR (KBr) v: 3031, 1758, 1592, 1265, 1211, 1063, 958, 848, $676 \mathrm{~cm}^{-1}$; MS (EI, $\left.70 \mathrm{eV}\right) \mathrm{m} / z(\%): 310.0$ $\left(\left[\mathrm{M}^{+\cdot}+2\right], 8\right), 308.0\left(\left[\mathrm{M}^{+\cdot}\right], 24\right), 183.1\left([\mathrm{M}+2-\mathrm{I}]^{+}, 34\right)$, $181.1\left([\mathrm{M}-\mathrm{I}]^{+}, 88\right), 169.0\left(\left[\mathrm{M}+2-\mathrm{CH}_{2} \mathrm{I}\right]^{+}, 39\right), 167.0$ $\left(\left[\mathrm{M}-\mathrm{CH}_{2} \mathrm{I}\right]^{+}, 100\right), 139.0\left(\left[p-\mathrm{ClC}_{6} \mathrm{H}_{4} \mathrm{CO}\right]^{+}, 24\right), 138.2$ $\left(\left[\mathrm{M}-\mathrm{ICH}_{2} \mathrm{CHO}\right]^{+}, 8\right), 127.0\left(\left[\mathrm{I}^{+}\right], 19\right), 113.0\left(\left[p-\mathrm{ClC}_{6}-\right.\right.$ $\left.\left.\mathrm{H}_{4}+2\right]^{+}, 7\right), 111.0\left(\left[p-\mathrm{ClC}_{6} \mathrm{H}_{4}\right]^{+}, 20\right)$. Anal. calcd for $\mathrm{C}_{9} \mathrm{H}_{6} \mathrm{ClIO}_{2}$ : C 35.04, $\mathrm{H} 1.96, \mathrm{Cl} 11.49$, I 41.14; found $\mathrm{C}$ 35.01, H 1.96, Cl 11.50, I 41.17.

3-碘甲基-5-溴异苯并呋喃-1(3H)-酮(7e)：淡黄色固 体. ${ }^{1} \mathrm{H}$ NMR $\left(400 \mathrm{MHz}, \mathrm{CDCl}_{3}\right) \delta: 7.80 \sim 7.73(\mathrm{~m}, 3 \mathrm{H}$, ArH), $5.46(\mathrm{t}, J=5.2 \mathrm{~Hz}, 1 \mathrm{H}, \mathrm{CH}), 3.62(\mathrm{dd}, J=10.8,5.2$ $\left.\mathrm{Hz}, 1 \mathrm{H}, \mathrm{CH}_{2}\right), 3.58$ (dd, $\left.J=11.2,4.8 \mathrm{~Hz}, 1 \mathrm{H}, \mathrm{CH}_{2}\right) ;{ }^{13} \mathrm{C}$ NMR (100 MHz, $\left.\mathrm{CDCl}_{3}\right) \delta: 168.29,149.92,133.68$, $129.61,127.17,125.77,125.59,77.88,4.89$; IR (KBr) $v$ : 3034, 1757, 1421, 1306, 1211, 1068, $674 \mathrm{~cm}^{-1}$; MS (EI, $70 \mathrm{eV}) \mathrm{m} / z(\%): 354.0\left(\left[\mathrm{M}^{+\cdot}+2\right], 27\right), 352.0\left(\left[\mathrm{M}^{+\cdot}\right], 27\right)$, $227.0\left([\mathrm{M}+2-\mathrm{I}]^{+}, 83\right), 225.0\left([\mathrm{M}-\mathrm{I}]^{+}, 79\right), 213.0([\mathrm{M}+$ $\left.\left.2-\mathrm{CH}_{2} \mathrm{I}\right]^{+}, 100\right), 211.0\left(\left[\mathrm{M}-\mathrm{CH}_{2} \mathrm{I}\right]^{+}, 96\right), 185.0$ $\left(\left[p-\mathrm{BrC}_{6} \mathrm{H}_{4} \mathrm{CO}+2\right]^{+}, 18\right), 183.0\left(\left[p-\mathrm{BrC}_{6} \mathrm{H}_{4} \mathrm{CO}\right]^{+}, 19\right)$, $182.1\left(\left[\mathrm{M}-\mathrm{ICH}_{2} \mathrm{CHO}\right]^{+}\right.$, 5), $155.0\left(\left[p-\mathrm{BrC}_{6} \mathrm{H}_{4}\right]^{+}, 16\right)$, $127.0\left(\left[\mathrm{I}^{+}\right], 14\right), 104.0\left(\left[\mathrm{M}-\mathrm{Br}-\mathrm{CH}_{2} \mathrm{I}-\mathrm{CO}\right]^{+\cdot}, 4\right)$. Anal. calcd for $\mathrm{C}_{9} \mathrm{H}_{6} \mathrm{BrIO}_{2}$ : C 30.63, H 1.71, Br 22.64, I 35.96; found C 30.60, H 1.71, Br 22.66, I 35.99.

3-碘甲基-6-溴异苯并呋喃-1(3H)-酮(7f)：淡黄色固 体. ${ }^{1} \mathrm{H}$ NMR (400 MHz, $\left.\mathrm{CDCl}_{3}\right) \delta: 8.06(\mathrm{~d}, J=1.6 \mathrm{~Hz}, 1 \mathrm{H}$, ArH), $7.84 \sim 7.82(\mathrm{~m}, 1 \mathrm{H}, \operatorname{ArH}), 7.51(\mathrm{~d}, J=8.4 \mathrm{~Hz}, 1 \mathrm{H}$, ArH), 5.45 (t, $J=5.2 \mathrm{~Hz}, 1 \mathrm{H}, \mathrm{CH}), 3.62(\mathrm{dd}, J=11.2,5.6$ $\left.\mathrm{Hz}, 1 \mathrm{H}, \mathrm{CH}_{2}\right), 3.58\left(\mathrm{dd}, J=8.4,2.0 \mathrm{~Hz}, 1 \mathrm{H}, \mathrm{CH}_{2}\right) ;{ }^{13} \mathrm{C}$ NMR (100 MHz, DMSO- $\left.d_{6}\right) \delta: 167.72,148.17,137.33$, $128.39,127.36,124.99,122.78,78.41,8.99$; IR (KBr) $v$ : 3018, 1763, 1467, 1296, 1205, 1070, 950, 821, 627, 514 $\mathrm{cm}^{-1}$; MS (EI, $\left.70 \mathrm{eV}\right) \mathrm{m} / z(\%): 354.0\left(\left[\mathrm{M}^{+\cdot}+2\right], 22\right), 352.0$ $\left(\left[\mathrm{M}^{+\cdot}\right], 22\right), 227.0\left([\mathrm{M}+2-\mathrm{I}]^{+}, 68\right), 225.0\left([\mathrm{M}-\mathrm{I}]^{+}, 63\right)$, $213.0\left(\left[\mathrm{M}+2-\mathrm{CH}_{2} \mathrm{I}\right]^{+}, 100\right), 211.0\left(\left[\mathrm{M}-\mathrm{CH}_{2} \mathrm{I}\right]^{+}, 93\right)$, $185.0\left(\left[m-\mathrm{BrC}_{6} \mathrm{H}_{4} \mathrm{CO}+2\right]^{+}, 18\right), 183.0\left(\left[m-\mathrm{BrC}_{6} \mathrm{H}_{4} \mathrm{CO}\right]^{+}\right.$, 19), $182.1\left(\left[\mathrm{M}-\mathrm{ICH}_{2} \mathrm{CHO}\right]^{+\cdot}, 5\right), 155.0\left(\left[m-\mathrm{BrC}_{6} \mathrm{H}_{4}\right]^{+}\right.$, 16), $127.0\left(\left[\mathrm{I}^{+}\right], 14\right), 104.0\left(\left[\mathrm{M}-\mathrm{Br}-\mathrm{CH}_{2} \mathrm{I}-\mathrm{CO}\right]^{+\cdot}, 4\right)$. Anal. calcd for $\mathrm{C}_{9} \mathrm{H}_{6} \mathrm{BrIO}_{2}$ : C 30.63, $\mathrm{H} \mathrm{1.71,} \mathrm{Br} 22.64$, I 35.96; found C 30.60, H 1.71, Br 22.66, I 36.00.

2.2 .43 -溴甲基异苯并呋喃-1(3H)-酮及其衍生物 8 合 成通法

在 $50 \mathrm{~mL}$ 三口烧瓶中加 $2 \mathrm{mmol}$ 化合物 $\mathbf{6}$, 加 $10 \mathrm{~mL}$ DMF 溶解，搅拌下加 $427 \mathrm{mg}(2.4 \mathrm{mmol}) \mathrm{NBS}$, 氮气保 护下, 室温反应 $6 \mathrm{~h}, \mathrm{TLC}$ 跟踪反应进程[展开剂: $V($ 乙酸 乙酯) $: V($ 石油醚 $)=1: 4]$. 向反应液加 $50 \mathrm{~mL}$ 水, 充分 搅拌后, 用乙醚 $(30 \mathrm{~mL} \times 3)$ 萃取, 有机层分别用饱和亚 硫酸氢钠溶液、碳酸氢钠溶液和食盐水 $(25 \mathrm{~mL} \times 2)$ 洗涤, 无水硫酸镁干燥，减压旋蒸，残余物真空干燥得化合物 8.

3-溴甲基异苯并呋喃-1(3H)-酮 $(\mathbf{8 a})^{[18]}$ : 白色固体， 产率 $89.8 \%$. m.p. $61.5 \sim 61.9{ }^{\circ} \mathrm{C}$ (文献值 ${ }^{[18]} 60.2 \sim$ $\left.62.2{ }^{\circ} \mathrm{C}\right) ;{ }^{1} \mathrm{H}$ NMR $\left(400 \mathrm{MHz}, \mathrm{CDCl}_{3}\right) \delta: 7.94(\mathrm{~d}, J=7.6$ $\mathrm{Hz}, 1 \mathrm{H}, \mathrm{ArH}), 7.73$ (t, J=7.6 Hz, 1H, ArH), 7.65 7.59 $(\mathrm{m}, 2 \mathrm{H}, \mathrm{ArH}), 5.70$ (t, $J=5.2 \mathrm{~Hz}, 1 \mathrm{H}, \mathrm{CH}), 3.77$ (d, $J=5.2$ $\left.\mathrm{Hz}, 1 \mathrm{H}, \mathrm{CH}_{2}\right)$.

3-溴甲基-4-溴异苯并呋喃-1(3H)-酮 $(8 \mathbf{b})$ : 白色固体, 产率 89.2\%. m.p. 119.9 120.3 ${ }^{\circ} \mathrm{C} ;{ }^{1} \mathrm{H}$ NMR (400 MHz, $\left.\mathrm{CDCl}_{3}\right) \delta: 7.82 \sim 7.32(\mathrm{~m}, 3 \mathrm{H}, \mathrm{ArH}), 5.67(\mathrm{t}, J=5.2 \mathrm{~Hz}$, $1 \mathrm{H}, \mathrm{CH}$ ), 3.79 (dd, $\left.J=11.2,4.4 \mathrm{~Hz}, 1 \mathrm{H}, \mathrm{CH}_{2}\right), 3.74$ (dd, $\left.J=10.8,5.6 \mathrm{~Hz}, 1 \mathrm{H}, \mathrm{CH}_{2}\right) ;{ }^{13} \mathrm{C} \mathrm{NMR}\left(100 \mathrm{MHz}, \mathrm{CDCl}_{3}\right)$ $\delta: 168.45,148.86,133.67,129.61,127.13,125.97,125.46$, 77.92, 31.78; IR (KBr) v: 1764, 1419, 1303, 1209, 1054, 973, 674, $618 \mathrm{~cm}^{-1}$; MS (EI, $\left.70 \mathrm{eV}\right) \mathrm{m} / z$ (\%): 307.9 $\left(\left[\mathrm{M}^{+\cdot}+4\right], 8\right), 305.9\left(\left[\mathrm{M}^{+\cdot}+2\right], 16\right), 304.0\left(\left[\mathrm{M}^{+\cdot}\right], 8\right)$, $213.0\left(\left[\mathrm{M}+2-\mathrm{CH}_{2} \mathrm{Br}\right]^{+}, 100\right), 211.0\left(\left[\mathrm{M}-\mathrm{CH}_{2} \mathrm{Br}\right]^{+}, 90\right)$, $185.0\left(\left[m-\mathrm{BrC}_{6} \mathrm{H}_{4} \mathrm{CO}+2\right]^{+}, 14\right), 183.0\left(\left[m-\mathrm{BrC}_{6} \mathrm{H}_{4} \mathrm{CO}\right]^{+}\right.$, 15), $157.0\left(\left[m-\mathrm{BrC}_{6} \mathrm{H}_{4}+2\right]^{+}, 10\right), 155.0\left(\left[m-\mathrm{BrC}_{6} \mathrm{H}_{4}\right]^{+}\right.$, 
11), $104.0\left(\left[\mathrm{M}-\mathrm{Br}-\mathrm{CH}_{2} \mathrm{Br}-\mathrm{CO}\right]^{+*}\right.$, 3). Anal. calcd for $\mathrm{C}_{9} \mathrm{H}_{6} \mathrm{Br}_{2} \mathrm{O}_{2}$ : C 35.33, H 1.98, $\mathrm{Br} 52.23$; found C 35.30, H 1.98, $\mathrm{Br} 52.27$.

3-溴甲基-4-硝基异苯并呋喃-1(3H)-酮(8c)：白色固 体, 产率 82.9\%. m.p. 104.6 106.5 ${ }^{\circ} \mathrm{C} ;{ }^{1} \mathrm{H}$ NMR $(400$ $\left.\mathrm{MHz}, \mathrm{CDCl}_{3}\right) \delta: 8.57 \sim 8.51$ (m, $\left.1 \mathrm{H}, \mathrm{ArH}\right), 8.30$ (d, $J=7.6$ $\mathrm{Hz}, 1 \mathrm{H}, \mathrm{ArH}), 7.87$ (t, $J=8.0 \mathrm{~Hz}, 1 \mathrm{H}, \mathrm{ArH}), 6.34$ (t, $J=2.8$ $\mathrm{Hz}, 1 \mathrm{H}, \mathrm{CH}), 4.23\left(\mathrm{dd}, J=12.0,3.2 \mathrm{~Hz}, 1 \mathrm{H}, \mathrm{CH}_{2}\right), 4.11$ (dd, $\left.J=12.0,2.8 \mathrm{~Hz}, 1 \mathrm{H}, \mathrm{CH}_{2}\right) ;{ }^{13} \mathrm{C}$ NMR $(100 \mathrm{MHz}$, $\left.\mathrm{CDCl}_{3}\right) \delta: 166.98,141.70,136.63,131.88,130.86,130.52$, 129.56, 79.31, 31.74; IR (KBr) v: 3096, 1779, 1466, 1300, 1194, 1058, 960, $792 \mathrm{~cm}^{-1}$; MS (EI, $\left.70 \mathrm{eV}\right) \mathrm{m} / \mathrm{z}(\%): 274.0$ $\left(\left[\mathrm{M}^{+\cdot}+3\right], 28\right), 273.0\left(\left[\mathrm{M}^{+\cdot}+2\right], 3\right), 272.0\left(\left[\mathrm{M}^{+\cdot}+1\right], 28\right)$, $192.1\left([\mathrm{M}-\mathrm{Br}]^{+}, 44\right), 178.1\left(\left[\mathrm{M}-\mathrm{CH}_{2} \mathrm{Br}\right]^{+}, 100\right), 149.1$ $\left(\left[\mathrm{M}-\mathrm{BrCH}_{2} \mathrm{CHO}\right]^{+}, 2\right), 104.1\left(\left[\mathrm{M}-\mathrm{NO}_{2}-\mathrm{CH}_{2} \mathrm{Br}-\right.\right.$ $\mathrm{CO}]^{+*}$, 38). Anal. calcd for $\mathrm{C}_{9} \mathrm{H}_{6} \mathrm{BrNO}_{4}$ : C 39.73, H 2.22, Br 29.37, N 5.15; found C 39.70, H 2.22, Br 29.39, N 5.16.

3-溴甲基-5-氯异苯并呋喃-1(3H)-酮 (8d): 白色固体, 产率 71.0\%. m.p. $114.3 \sim 116.8{ }^{\circ} \mathrm{C} ;{ }^{1} \mathrm{H}$ NMR $(400 \mathrm{MHz}$, $\left.\mathrm{CDCl}_{3}\right) \delta: 7.86(\mathrm{~d}, J=8.0 \mathrm{~Hz}, 1 \mathrm{H}, \mathrm{ArH}), 7.65(\mathrm{~s}, 1 \mathrm{H}$, ArH ), 7.58 (d, $J=8.0 \mathrm{~Hz}, 1 \mathrm{H}, \mathrm{ArH}), 5.67$ (t, $J=4.8 \mathrm{~Hz}$, $1 \mathrm{H}, \mathrm{CH}), 3.79\left(\mathrm{dd}, J=11.2,4.4 \mathrm{~Hz}, 1 \mathrm{H}, \mathrm{CH}_{2}\right), 3.73(\mathrm{dd}$, $\left.J=10.4,5.6 \mathrm{~Hz}, 1 \mathrm{H}, \mathrm{CH}_{2}\right) ;{ }^{13} \mathrm{C} \mathrm{NMR}\left(100 \mathrm{MHz}, \mathrm{CDCl}_{3}\right)$ $\delta: 168.32,148.77,141.12,130.84,127.06,125.01,123.01$, 77.95, 31.77; IR (KBr) v: 3029, 1758, 1459, 1365, 1210, 1077, 984, 845, $642 \mathrm{~cm}^{-1}$; MS (EI, $70 \mathrm{eV}$ ) $\mathrm{m} / \mathrm{z}(\%): 264.0$ $\left(\left[\mathrm{M}^{+\cdot}+4\right], 5\right), 262.0\left(\left[\mathrm{M}^{+\cdot}+2\right], 20\right), 260.0\left(\left[\mathrm{M}^{+\cdot}\right], 15\right)$, $169.0\left(\left[\mathrm{M}+2-\mathrm{CH}_{2} \mathrm{Br}\right]^{+}, 80\right), 167.1\left(\left[\mathrm{M}-\mathrm{CH}_{2} \mathrm{Br}\right]^{+}, 100\right)$, $139.0\left(\left[p-\mathrm{ClC}_{6} \mathrm{H}_{4} \mathrm{CO}\right]^{+}, 30\right), 113.0\left(\left[p-\mathrm{ClC}_{6} \mathrm{H}_{4}+2\right]^{+}, 9\right)$, $111.0\left(\left[p-\mathrm{ClC}_{6} \mathrm{H}_{4}\right]^{+}\right.$, 26). Anal. calcd for $\mathrm{C}_{9} \mathrm{H}_{6} \mathrm{BrClO}_{2}$ : C 41.34, H 2.31, Br 30.56, Cl 13.56; found C 41.31, H 2.31, Br 30.59, Cl 13.57.

3-溴甲基-5-溴异苯并呋喃-1(3H)-酮 $(8 \mathbf{e})$ : 白色固体, 产率 94.5\%. m.p. 109.9 110.5 ${ }^{\circ} \mathrm{C} ;{ }^{1} \mathrm{H}$ NMR $(400 \mathrm{MHz}$, $\left.\mathrm{CDCl}_{3}\right) \delta: 7.82 \sim 7.79(\mathrm{~m}, 2 \mathrm{H}, \mathrm{ArH}), 7.74(\mathrm{~d}, J=8.4 \mathrm{~Hz}$, $1 \mathrm{H}, \mathrm{ArH}), 5.67$ (t, $J=5.2 \mathrm{~Hz}, 1 \mathrm{H}, \mathrm{CH}), 3.78$ (dd, $J=11.2$, $\left.4.4 \mathrm{~Hz}, 1 \mathrm{H}, \mathrm{CH}_{2}\right), 3.73\left(\mathrm{dd}, J=11.2,6.0 \mathrm{~Hz}, 1 \mathrm{H}, \mathrm{CH}_{2}\right) ;{ }^{13} \mathrm{C}$ NMR $\left(100 \mathrm{MHz}, \mathrm{CDCl}_{3}\right) \delta: 168.55,148.88,133.70$, 129.66, 127.17, $125.99,125.46,77.96,31.77$; IR (KBr) $v$ : 3062, 1762, 1466, 1269, 1210, 1063, 974, $835 \mathrm{~cm}^{-1}$; MS (EI, $70 \mathrm{eV}) \mathrm{m} / \mathrm{z}(\%): 308.0\left(\left[\mathrm{M}^{++}+4\right], 8\right), 306.0\left(\left[\mathrm{M}^{++}+2\right]\right.$, 16), $304.0\left(\left[\mathrm{M}^{+\cdot}\right], 8\right), 213.0\left(\left[\mathrm{M}+2-\mathrm{CH}_{2} \mathrm{Br}\right]^{+}, 100\right)$, $211.0\left(\left[\mathrm{M}-\mathrm{CH}_{2} \mathrm{Br}\right]^{+}, 92\right), 185.0\left(\left[p-\mathrm{BrC}_{6} \mathrm{H}_{4} \mathrm{CO}+2\right]^{+}, 14\right)$, $183.0\left(\left[p-\mathrm{BrC}_{6} \mathrm{H}_{4} \mathrm{CO}\right]^{+}, 15\right), 157.0\left(\left[p-\mathrm{BrC}_{6} \mathrm{H}_{4}+2\right]^{+}, 10\right)$, $155.0\left(\left[p-\mathrm{BrC}_{6} \mathrm{H}_{4}\right]^{+}, 12\right), 104.1\left(\left[\mathrm{M}-\mathrm{Br}-\mathrm{CH}_{2} \mathrm{Br}-\mathrm{CO}\right]^{+}\right.$,
3). Anal. calcd for $\mathrm{C}_{9} \mathrm{H}_{6} \mathrm{Br}_{2} \mathrm{O}_{2}$ : C 35.33, H 1.98, $\mathrm{Br} 52.23$; found $\mathrm{C} 35.29, \mathrm{H}$ 1.98, $\mathrm{Br} 52.28$.

3-溴甲基-6-溴异苯并呋喃-1(3H)-酮(8f): 白色固体, 产率 97.9\%. m.p. 129.4 130.9 ${ }^{\circ} \mathrm{C} ;{ }^{1} \mathrm{H}$ NMR $(400 \mathrm{MHz}$, $\left.\mathrm{CDCl}_{3}\right) \delta: 8.07$ (s, $\left.1 \mathrm{H}, \mathrm{ArH}\right), 7.84(\mathrm{~d}, J=8.4 \mathrm{~Hz}, 1 \mathrm{H}, \mathrm{ArH})$, $7.53(\mathrm{~d}, J=8.4 \mathrm{~Hz}, 1 \mathrm{H}, \mathrm{ArH}), 5.66(\mathrm{t}, J=5.2 \mathrm{~Hz}, 1 \mathrm{H}, \mathrm{CH})$, $3.78\left(\mathrm{dd}, J=11.2,4.4 \mathrm{~Hz}, 1 \mathrm{H}, \mathrm{CH}_{2}\right), 3.72(\mathrm{dd}, J=11.2,6.0$ $\left.\mathrm{Hz}, 1 \mathrm{H}, \mathrm{CH}_{2}\right) ;{ }^{13} \mathrm{C}$ NMR $\left(100 \mathrm{MHz}, \mathrm{CDCl}_{3}\right) \delta: 167.86$, $145.82,137.43,128.87,128.55,124.15,124.06,78.45$, 31.79; IR (KBr) v: 3057, 1764, 1471, 1296, 1200, 1074, 962, 826.8, $631 \mathrm{~cm}^{-1}$; MS (EI, $70 \mathrm{eV}$ ) $\mathrm{m} / \mathrm{z}(\%): 308.0$ $\left(\left[\mathrm{M}^{+\cdot}+4\right], 8\right), 306.0\left(\left[\mathrm{M}^{+\cdot}+2\right], 17\right), 304.0\left(\left[\mathrm{M}^{+\cdot}\right], 9\right)$, $213.0\left(\left[\mathrm{M}+2-\mathrm{CH}_{2} \mathrm{Br}\right]^{+}, 100\right), 211.0\left(\left[\mathrm{M}-\mathrm{CH}_{2} \mathrm{Br}\right]^{+}, 96\right)$, $185.0\left(\left[m-\mathrm{BrC}_{6} \mathrm{H}_{4} \mathrm{CO}+2\right]^{+}, 16\right), 183.0\left(\left[m-\mathrm{BrC}_{6} \mathrm{H}_{4} \mathrm{CO}\right]^{+}\right.$, 17), $157.0\left(\left[m-\mathrm{BrC}_{6} \mathrm{H}_{4}+2\right]^{+}, 12\right), 155.0\left(\left[m-\mathrm{BrC}_{6} \mathrm{H}_{4}\right]^{+}\right.$, 13), $104.0\left(\left[\mathrm{M}-\mathrm{Br}-\mathrm{CH}_{2} \mathrm{Br}-\mathrm{CO}\right]^{+\cdot}\right.$, 4). Anal. calcd for $\mathrm{C}_{9} \mathrm{H}_{6} \mathrm{Br}_{2} \mathrm{O}_{2}$ : C 35.33, H 1.98, Br 52.23; found C 35.28, H 1.98, Br 52.28.

2.2.5 3-亚甲基异苯并呋喃-1 (3H) - 酮及其衍生物9 合 成通法

在 $100 \mathrm{~mL}$ 三颈瓶中加 $1 \mathrm{mmol}$ 化合物 7 或者 8 , 加 $30 \mathrm{~mL}$ 苯溶解, 搅拌下加 $278 \mathrm{mg}$ (1.83 mmol) 1,8 -二氮杂 二环 [5.4.0]十一碳-7-烯(DBU), 加热回流 $2 \mathrm{~h}, \mathrm{TLC}$ 检测 反应进程 [展开剂: $V$ (乙酸乙酯) $: V$ (石油醚 $)=1: 4]$ ] 反 应结束后, 室温冷却, 加 $15 \mathrm{~mL}$ 饱和氯化铵溶液, 充分 搅拌后, 用乙醚 $(25 \mathrm{~mL} \times 3)$ 萃取, 有机层用饱和食盐水 $(20 \mathrm{~mL} \times 2)$ 洗涤, 无水硫酸镁干燥, 滤液旋至浓溶液. 柱层析分离 $[V$ (乙酸乙酯) $:$ (石油醚 $)=1: 1]$, 层析液旋 蒸至干，真空干燥得化合物 $\mathbf{9}$.

3-亚甲基异苯并呋喃-1(3H)-酮 $(9 \mathbf{a})^{[31]}$ : 白色固体, 产率 98.6\% (-HI), 90.5\% (-HBr). m.p. $55.8 \sim 56.3{ }^{\circ} \mathrm{C}$ (文献值 ${ }^{[32]} 54 \sim 55{ }^{\circ} \mathrm{C}$ ); ${ }^{1} \mathrm{H}$ NMR (400 MHz, DMSO- $\left.d_{6}\right) \delta$ : 8.09 (d, $J=4.0 \mathrm{~Hz}, 1 \mathrm{H}, \mathrm{ArH}), 7.95 \sim 7.87$ (m, 2H, ArH), $7.71(\mathrm{t}, J=8.0 \mathrm{~Hz}, 1 \mathrm{H}, \mathrm{ArH}), 5.62(\mathrm{~d}, J=4.0 \mathrm{~Hz}, 1 \mathrm{H},=$ $\left.\mathrm{CH}_{2}\right), 5.32\left(\mathrm{~d}, J=4.0 \mathrm{~Hz}, 1 \mathrm{H},=\mathrm{CH}_{2}\right)$.

3 -亚甲基-4-溴异苯并呋喃-1(3H)-酮 $(\mathbf{9 b})^{[8]}$ : 白色固 体, 产率 $99.1 \%(-\mathrm{HI}), 91.6 \%$ (- HBr). m.p. 77.9 $79.1{ }^{\circ} \mathrm{C}$ (文献值 ${ }^{[8]} 78.8 \sim 80.2{ }^{\circ} \mathrm{C}$ ); ${ }^{1} \mathrm{H}$ NMR $(500 \mathrm{MHz}$, $\left.\mathrm{CDCl}_{3}\right) \delta: 7.92 \sim 7.90(\mathrm{~m}, 2 \mathrm{H}, \mathrm{ArH}), 7.45(\mathrm{t}, J=7.5 \mathrm{~Hz}$, $1 \mathrm{H}, \mathrm{ArH}), 6.06\left(\mathrm{~d}, J=3.0 \mathrm{~Hz}, 1 \mathrm{H},=\mathrm{CH}_{2}\right), 5.42(\mathrm{~d}, J=$ $3.0 \mathrm{~Hz}, 1 \mathrm{H},=\mathrm{CH}_{2}$ ).

3-亚甲基-4-硝基异苯并呋喃-1(3H)-酮 $(\mathbf{9 c})^{[8]}$ : 白色 固体, 产率 $92.9 \%(-\mathrm{HI}), 89.6 \%(-\mathrm{HBr})$. m.p. 101.5 $101.9{ }^{\circ} \mathrm{C}$ (文献值 ${ }^{[8]} 101.2 \sim 102.3{ }^{\circ} \mathrm{C}$ ); ${ }^{1} \mathrm{H}$ NMR $(500$ $\left.\mathrm{MHz}, \mathrm{CDCl}_{3}\right) \delta: 8.46(\mathrm{~d}, J=8.0 \mathrm{~Hz}, 1 \mathrm{H}, \mathrm{ArH}), 8.27$ (d, $J=$ 
$7.5 \mathrm{~Hz}, 1 \mathrm{H}, \mathrm{ArH}), 7.81$ (t, $J=7.5 \mathrm{~Hz}, 1 \mathrm{H}, \mathrm{ArH}), 6.20$ (d, $\left.J=3.0 \mathrm{~Hz}, 1 \mathrm{H},=\mathrm{CH}_{2}\right), 5.78\left(\mathrm{~d}, J=3.0 \mathrm{~Hz}, 1 \mathrm{H},=\mathrm{CH}_{2}\right)$.

3 -亚甲基-5-氯异苯并呋喃-1(3H)-酮 $(9 d)^{[9]}$ : 白色固 体, 产率 98.6\% (- $\mathrm{HI}), 92.9 \%(-\mathrm{HBr})$. m.p. 107.5 $112.4{ }^{\circ} \mathrm{C}$ (文献值 ${ }^{[9]} 107.5 \sim 112.4{ }^{\circ} \mathrm{C}$ ); ${ }^{1} \mathrm{H}$ NMR (400 $\left.\mathrm{MHz}, \mathrm{CDCl}_{3}\right) \delta: 7.85(\mathrm{~d}, J=8.0 \mathrm{~Hz}, 1 \mathrm{H}, \mathrm{ArH}), 7.70(\mathrm{~s}, 1 \mathrm{H}$, ArH), 7.55 (dd, $J=8.0,4.0 \mathrm{~Hz}, 1 \mathrm{H}, \mathrm{ArH}), 5.29$ (d, $J=4.0$ $\left.\mathrm{Hz}, 1 \mathrm{H},=\mathrm{CH}_{2}\right), 5.24\left(\mathrm{~d}, J=4.0 \mathrm{~Hz}, 1 \mathrm{H},=\mathrm{CH}_{2}\right) ;{ }^{13} \mathrm{C}$ NMR (100 MHz, $\left.\mathrm{CDCl}_{3}\right) \delta: 165.70,150.64,141.32$, $140.45,131.13,126.51,123.46,120.90$.

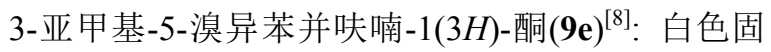
体, 产率 99.6\% (- $\mathrm{HI}), 85.6 \%$ (- $\mathrm{HBr})$. m.p. 97.5 $98.6{ }^{\circ} \mathrm{C}$ (文献值 ${ }^{[8]} 98.5 \sim 99.2{ }^{\circ} \mathrm{C}$ ); ${ }^{1} \mathrm{H}$ NMR $(500 \mathrm{MHz}$, $\left.\mathrm{CDCl}_{3}\right) \delta: 7.88(\mathrm{~s}, 1 \mathrm{H}, \mathrm{ArH}), 7.78(\mathrm{~d}, J=8.5 \mathrm{~Hz}, 1 \mathrm{H}, \mathrm{ArH})$, $7.72 \sim 7.71(\mathrm{~m}, 1 \mathrm{H}, \mathrm{ArH}), 5.29(\mathrm{~d}, J=3.0 \mathrm{~Hz}, 1 \mathrm{H}$, = $\left.\mathrm{CH}_{2}\right), 5.24\left(\mathrm{~d}, J=3.5 \mathrm{~Hz}, 1 \mathrm{H},=\mathrm{CH}_{2}\right)$.

3 -亚甲基-6-溴异苯并呋喃-1(3H)-酮(9f $)^{[8]}$ : 白色固 体, 产率 99.9\% (- $\mathrm{HI}), 94.1 \%$ (- $\mathrm{HBr})$. m.p. 94.5 $95.3{ }^{\circ} \mathrm{C}$ (文献值 ${ }^{[8]} 95.7 \sim 95.8{ }^{\circ} \mathrm{C}$ ); ${ }^{1} \mathrm{H}$ NMR (400 MHz, $\left.\mathrm{CDCl}_{3}\right) \delta: 8.05(\mathrm{~d}, J=1.2 \mathrm{~Hz}, 1 \mathrm{H}, \mathrm{ArH}), 7.84$ (dd, $J=8.0$, $1.6 \mathrm{~Hz}, 1 \mathrm{H}, \mathrm{ArH}), 7.60$ (d, J=8.0 Hz, 1H, ArH), 5.30 (d, $\left.J=4.0 \mathrm{~Hz}, 1 \mathrm{H},=\mathrm{CH}_{2}\right), 5.25\left(\mathrm{~d}, J=4.0 \mathrm{~Hz}, 1 \mathrm{H},=\mathrm{CH}_{2}\right)$.

辅助材料(Supporting Information) 合成化合物的核 磁共振氢谱. 这些材料可以免费从本刊网站(http://siocjournal.cn/)上下载.

\section{References}

[1] Yoshiyasu, F.; Fuzen, O.; Sensuke, K.; Yoshio, O. JP 61007267, 1986 [Chem. Abstr. 1986, 105, 85185y].

[2] Zhu, Z.-Y.; Yang, Y.; Tao, Y.; Wu, Y.-H.; Wu, G.-Q. J. Univ. Sci. Technol. China 1987, 17, 336 (in Chinese).

(朱胄远, 杨云, 陶荣, 莫东荣, 伍越环, 吴恭谦, 中国科学技术 大学学报, 1987, 17, 336.)

[3] Wu, Y.-H.; Zhu, Z.-Y.; Xi, Y.; Liu, L.; Wu, G.-Q.; Zhang, C. J. Univ. Sci. Technol. China 1991, 21, 163 (in Chinese). (伍越环, 朱胄远, 席英, 刘雷, 吴恭谦, 张超, 中国科学技术大 学学报, 1991, 21, 163.)

[4] Mares, D.; Bonora, A.; Sacchetti, G.; Rubini, M.; Romagnoli, C. Cell Biol. Int. 1997, 21, 397.

[5] Sun, X.-M.; Mao, Y.-H. J. Anhui Univ., Nat. Sci. 1991, 1, 65 (in Chinese). (孙熊鸣, 毛羽华, 安徽大学学报(自然科学版), 1991, 1, 65.)

[6] Xi, M.-M.; Zhang, S.-Q.; Wang, X.-Y.; Fang, K.-Q.; Gu, Y. Int. J. Pharm. 2005, 298, 91.

[7] Kitamura, T.; Kawakami, Y.; Imagawa, T.; Kawanisi, M. Tetrahedron 1980, 36, 1183.
[8] Zhang, J.-Z.; Wu, J.; Wang, Y.-Z.; Zhao, D.-J.; Jia, H.-B. Chin. J. Org. Chem. 2012, 32, 2344 (in Chinese).

(张继振, 吴健, 王雅珍, 赵德建, 贾洪斌, 有机化学, 2012, 32, 2344.)

[9] Zheng, C.-Z.; Zhu, X.-X.; Zhao, D.-J.; Jia, H.-B.; Zhang, J.-Z. Chin. J. Org. Chem. 2014, 34, 1881 (in Chinese).

(郑纯智, 朱星星, 赵德建, 贾洪斌, 张继振, 有机化学, 2014, 34, 1881.)

[10] Li, Z.; Li, J.; Yang, N.; Chen, Y.; Zhou, Y.; Ji, X.; Zhang, L.; Wang, J.-F.; Xie, X.; Liu, H. J. Org. Chem. 2013, 78, 10802.

[11] Rambabu, D.; Kumar, G. P.; Kumar, B. D.; Kapavarapu, R.; Pal, M. Tetrahedron Lett. 2013, 54, 2989.

[12] Zhang, B.; Xu, M. H.; Lin, G. Q. Org. Lett. 2009, 11, 4712.

[13] Lácová, M.; Chovancová, J.; Veverková, E.; Toma, Š. Tetrahedron 1996, 52, 14995.

[14] Wu, J.; Zhang, J.-Z.; Wang, Y.-Z.; Jia, H.-B. Chem. Res. Appl. 2012, 24, 791 (in Chinese).

(吴健, 张继振, 王雅珍, 贾洪斌, 化学研究与应用, 2012，24, 791.)

[15] Li, S.-B.; Li, Y.; Li, Y.-L. J. Lanzhou Univ., Nat. Sci. 1993, 29, 256.

(李绍白, 李瀛, 李裕林, 兰州大学学报(自然科学版), 1993, 29, 256.)

[16] Mali, R. S.; Patil, S. R. Synth. Commun. 1990, 20, 167.

[17] Rengewitsch. Doklady Chem. 1962, 146, 787.

[18] Chen, J.; Zhou, L.; Tan, C. K; Yeung, Y. Y. J. Org. Chem. 2012, 77, 999.

[19] Judd, L. W.; Davis, A. P. Chem. Commun. (Cambridge, United Kingdom) 2010, 46, 2227.

[20] Laufer, S. A.; Ahrens, G. M.; Karcher, S. C.; Hering, J. S.; Niess, R. J. Med. Chem. 2006, 49, 7912.

[21] Ding, W. Y.; Pu, J. Q.; Zhang, C. M.; Cao, W. G. J. Chem. Soc., Perkin Trans. 1 1991, 1369.

[22] Wu, J. M.S. Thesis, Changzhou University, Changzhou, 2012 (in Chinese). (吴健, 硕士论文, 常州大学, 常州, 2012.)

[23] Soederberg, B. C.; Shriver, J. A.; Wallace, J. M. Org. Synth. 2003, 80,75 .

[24] Quagliato, D. A.; Andrate, P, M.; Fan, Y. US 20070203116, 2007 [Chem. Abstr. 2007, 147, 322990].

[25] Mejia-Oneto, J. M.; Padwa, A. Org. Lett. 2004, 6, 3241.

[26] Gao, C.-S.; Zhao, L.-C.; Chen, X.-D.; Fan, X.-F.; Xie, X.-H.; Zhou, P.-Y. Macromolecules 2007, 40, 5718.

[27] Kamata, M.; Yamashita, T.; Imaeda, T.; Tanaka, T.; Masada, S.; Kamaura, M.; Kasai, S.; Hara, R.; Sasaki, S.; Takekawa, S.; Asami, A.; Kaisho, T.; Suzuki, N.; Ashina, S.; Ogino, H.; Nakano, Y.; Nagisa, Y.; Kato, K.; Kato, K.; Ishihara, Y. J. Med. Chem. 2012, 55, 2353.

[28] Ishihara, Y.; Kamata, M.; Takekawa, S.; Suzuki, N.; Kato, K. WO 2003035624, 2003 [Chem. Abstr. 2003, 138, 353842].

[29] Cheng, Y.; Choquette, D.; Harmange, J.-C.; Tasker, A. US 20080161346, 2008 [Chem. Abstr. 2008, 149, 128760].

[30] Reich, S. H.; Melnick, M.; Pino, M. J.; Fuhry, M. A. M.; Trippe, A. J.; Appelt, K.; Davies II, J. F.; Wu, B.-W.; Musick, L. J. Med. Chem. 1996, 39, 2781.

[31] Shang, R.; Fu, Y.; Li, J. B.; Zhang, S. L.; Guo, Q. X.; Liu, L. J. Am. Chem. Soc. 2009, 131, 5738.

[32] Nishio, T. J. Chem. Soc., Perkin Trans. 1 1995, 561. 